\title{
Receptivity of a high-speed boundary layer to temperature spottiness
}

\author{
A. V. Fedorov ${ }^{1}$, A. A. Ryzhov ${ }^{2,1}$, V. G. Soudakov ${ }^{2,1} \dagger$ and S. V. Utyuzhnikov ${ }^{3,1}$ \\ ${ }^{1}$ Moscow Institute of Physics and Technology, Dolgoprudny, Moscow region, 141700, Russia \\ ${ }^{2}$ Central Aerohydrodynamic Institute, Zhukovsky, 140180, Russia \\ ${ }^{3}$ School of Mechanical, Aerospace and Civil Engineering, University of Manchester, Sackville Street, \\ Manchester M13 9PL, UK
}

(Received 15 March 2012; revised 28 November 2012; accepted 19 February 2013)

Two-dimensional direct numerical simulation (DNS) of the receptivity of a flat-plate boundary layer to temperature spottiness in the Mach 6 free stream is carried out. The influence of spottiness parameters on the receptivity process is studied. It is shown that the temperature spots propagating near the upper boundary-layer edge generate mode $\mathrm{F}$ inside the boundary layer. Further downstream mode $\mathrm{F}$ is synchronized with unstable mode S (Mack second mode) and excites the latter via the inter-modal exchange mechanism. Theoretical assessments of the mode $\mathrm{F}$ amplitude are made using the biorthogonal eigenfunction decomposition method. The DNS results agree with the theoretical predictions. If the temperature spots are initiated in the free stream and pass through the bow shock, the dominant receptivity mechanism is different. The spot-shock interaction leads to excitation of acoustic waves, which penetrate into the boundary layer and excite mode S. Numerical simulations show that this mechanism provides the instability amplitudes an order of magnitude higher than in the case of receptivity to the temperature spots themselves.

Key words: boundary-layer receptivity, boundary-layer stability, compressible flows

\section{Introduction}

Estimation of laminar-turbulent transition locations in the boundary layer is important for the development of hypersonic vehicles (Kimmel 2003). The first reason is that early transition leads to the increase of drag and hence the loss of the aircraft efficiency. The second is associated with the growth of wall heat fluxes that lead to the increase of the weight of thermal protection systems (TPSs). This motivates extensive experimental, theoretical and numerical studies of the transition at supersonic and hypersonic speeds. The progress made in the transition prediction methodology for high speeds is reviewed by Malik, Zang \& Bushnell (1990), Malik (1997) and Fedorov (2011). The success of transition prediction depends on a fundamental understanding of the relevant physical mechanisms. Despite considerable efforts many critical aspects of these mechanisms are still poorly understood especially at hypersonic speeds (Reshotko 2008). 
In a weak-disturbance environment and on aerodynamically smooth surfaces, the route leading to turbulence in the boundary-layer flow includes: receptivity, linear phase of instability evolution and nonlinear breakdown to turbulence. Receptivity refers to the mechanism by which free-stream disturbances generate unstable modes in the boundary layer. The receptivity process provides initial conditions for amplitudes, frequencies and phases of the boundary-layer modes. The linear phase is associated with the downstream growth of these modes. It is governed by the linear stability theory (LST) (Mack 1969). The nonlinear breakdown occurs when the disturbance amplitudes achieve a certain critical level. The receptivity process is still poorly understood, especially at high speeds. This prevents from developing of rational transition prediction methods, which couple the transition locus with characteristics of free-stream disturbances and avoid the empiricism of the $e^{N}$ method (Jaffe, Okamura \& Smith 1970; Malik et al. 1990).

There are three types of small free-stream disturbances: acoustic waves (fast and slow), entropy waves (temperature spottiness) and vorticity waves. Acoustics waves propagate at the speed of sound with respect to the flow, whereas vorticity and entropy waves travel with the mean flow speed. Free-stream acoustic waves dominate in conventional wind tunnel experiments due to acoustic radiation of the turbulent boundary layer on the wind tunnel walls. Free-stream entropy waves are related to variable temperature and density, while free-stream vorticity waves are associated with turbulence. Temperature spottiness and vorticity fluctuations dominate in flight where there are no external acoustic sources.

The receptivity of hypersonic boundary layers to free-stream acoustic waves was extensively studied theoretically, experimentally and numerically. The theoretical model of receptivity to acoustic disturbances interacting with the boundary-layer flow near the flat-plate leading edge was developed by Fedorov \& Khokhlov $(1991,2001)$ and Fedorov (2003) using asymptotic theory. This model shows that the receptivity process is associated with diffraction and scattering of acoustic waves in the leading edge vicinity. The boundary-layer mode excited near the leading edge by a fast acoustic wave can be referred as mode $\mathrm{F}$ and by a slow acoustic wave as mode $\mathrm{S}$. The theoretical predictions (Fedorov 2003) of receptivity coefficients agree well with the experimental data of Maslov et al. (2001), which were obtained in the Mach 6 wind tunnel. The direct numerical simulation (DNS) results of Ma \& Zhong (2001, 2003), Egorov, Fedorov \& Soudakov (2005, 2008), Malik \& Balakumar (2005) and Soudakov (2010) and other researchers agreed with the theory.

For high-speed flows, the asymptotic structure of the disturbances field and the receptivity region essentially differ from the case of subsonic flow analyzed by Goldstein (1983). For subsonic boundary layers the phase speed of free-stream disturbances is significantly different from the phase speed of Tollmien-Schlichting (TS) waves. A scale-conversion mechanism is needed to tune the time and length scales of external disturbances. Theoretical studies of Goldstein (1983, 1985), Ruban (1984) and Zhigulev \& Fedorov (1987) and the later investigations of Choudhari \& Streett (1990) and Choudhari (1994) showed that the scale conversion of acoustic waves can be achieved near the leading edge where the mean flow is non-parallel, in the vicinity of a surface imperfection or over a wavy wall or hump. The bilinear interaction between a pair of free-stream disturbances can also provide the scale conversion. Wu (1999) has elaborated on this mechanism by considering suitable convecting gusts, which interact with sound waves to produce a forcing that has the same time and length scales as those of TS waves in the vicinity of the neutral point. 
The receptivity to free-stream entropy and vortical disturbances has been investigated substantially less. Zhong (2001) carried out numerical simulations of receptivity to the entropy and vorticity waves (with zero angle of inclination) interacting with the boundary layer over a parabola. Ma \& Zhong (2005) performed DNSs of receptivity of the flat-plate boundary layer to entropy and vorticity waves with several positive angles of inclination.

Fedorov \& Tumin (2003) analyzed an initial value problem for a twodimensional wavepacket induced by a local two-dimensional disturbance in a hypersonic boundary layer. Characteristics of the wavepacket generated by an initial temperature spot were calculated. It was shown that the hypersonic boundary layer is receptive to vorticity/entropy disturbances in the region of synchronization between entropy/vorticity modes of temporal continuous spectrum and normal modes of discrete spectrum.

Schneider, Collicott \& Schmeisseur (2000), Schmeisseur, Schneider \& Collicott (2002) and Salyer, Collicott \& Schneider (2006) experimentally investigated disturbances induced by a laser beam near a cone at hypersonic flow conditions. It was shown that these disturbances have approximately Gaussian radial temperature distributions. The heat spot generates both acoustic and vorticity disturbances while transmitting through the shock. Huang \& Zhong (2010) carried out DNSs of a single entropy spot propagating past a sharp cone for the conditions of Boeing/AFOSR Mach-6 Quiet tunnel in Purdue University (Schneider et al. 2009).

Heitmann, Radespiel \& Knauss (2011) experimentally investigated the response of a Mach 6 hypersonic boundary layer on the cone to artificial disturbances. A high-power laser was used to generate expanding shock wave and a volume of heated gas. It was found that this forcing excited the second-mode wave train in the boundary layer. Heitmann \& Radespiel (2011) performed numerical simulations of this process using a self-similar explosion solution which mimics the laser generated spot.

In the foregoing DNS and experimental studies, free-stream entropy/vortical disturbances pass through the bow shock. The interaction between these disturbances and the shock wave leads to the excitation of all three kinds of disturbances behind the shock (Djakov 1957; McKenzie \& Westphal 1968) including acoustic waves. The boundary-layer instability can be excited by these acoustic waves or by entropy/vortical disturbances themselves. For proper modelling of the receptivity process, it is important to clarify basic features of these two roots and understand which of them is dominant. In the present paper this issue is addressed with the help of DNS and theoretical assessments. Two-dimensional DNS of flat-plate boundarylayer receptivity to temperature spottiness in Mach-6 free stream is carried out. The Navier-Stokes equations for two-dimensional unsteady compressible flow are solved using the modified algorithm (Egorov, Fedorov \& Soudakov 2006) in which the advection terms are approximated by a third-order weighted essentially non-oscillatory (WENO) scheme. This allows us to simulate evolution of small disturbances with a sufficient accuracy.

To study receptivity to the entropy fluctuations themselves, a temperature spottiness is introduced downstream of the bow shock. The temperature spots have a Gaussian distribution in the vertical direction and a sine distribution in the longitudinal direction. The spots are located slightly above the upper boundary-layer edge. They travel downstream and excite small disturbances in the boundary layer. The latter generate unstable waves related to the Mack second mode. Theoretical assessments of the initial amplitudes of excited instability are performed using the biorthogonal eigenfunction 
decomposition method (Zhigulev \& Tumin 1987; Fedorov \& Tumin 2003; Gaydos \& Tumin 2004). The DNS results are compared with the theoretical predictions.

The effects associated with the interaction of free-stream temperature spots with the bow shock are simulated numerically. The spot-shock interaction leads to the excitation of acoustic waves which, in turn, generate unstable disturbances of relatively high amplitude. This mechanism is compared with receptivity to the temperature spottiness induced downstream of the bow shock.

\section{Problem formulation and numerical method}

Viscous two-dimensional unsteady compressible flows are described by the Navier-Stokes equations. Numerical simulations are carried out for a flat plate with a sharp leading edge. The flow variables are made non-dimensional using the steadystate free-stream parameters (denoted by subscript ' $\infty$ '): $(u, v)=\left(u^{*}, v^{*}\right) / U_{\infty}^{*}$ are longitudinal and vertical components of the flow velocity, $p=p^{*} /\left(\rho_{\infty}^{*} U_{\infty}^{* 2}\right)$ is pressure, $\rho=\rho^{*} / \rho_{\infty}^{*}$ is density and $T=T^{*} / T_{\infty}^{*}$ is temperature. The non-dimensional coordinates are $(x, y)=\left(x^{*}, y^{*}\right) / L^{*}$ and time is $t=t^{*} U_{\infty}^{*} / L^{*}$, where $U_{\infty}^{*}$ is the free-stream velocity and $L^{*}$ is the plate length. Hereinafter, asterisks denote dimensional variables. The fluid is assumed to be a perfect gas with the specific heat ratio $\gamma=1.4$ and Prandtl number $\operatorname{Pr}=0.72$. Calculations are performed for the Mach number 6 and the Reynolds number $\operatorname{Re}_{L}=\rho_{\infty}^{*} U_{\infty}^{*} L^{*} / \mu_{\infty}^{*}=2 \times 10^{6}$, where $\mu_{\infty}^{*}$ is the free-stream dynamic viscosity coefficient. The viscosity-temperature dependence is approximated by the power law $\mu^{*} / \mu_{\infty}^{*}=\left(T^{*} / T_{\infty}^{*}\right)^{0.7}$. The second viscosity is assumed to be zero. The plate has a nominally sharp leading edge, i.e. the leading-edge radius is so small that the bluntness-induced entropy layer does not affect receptivity and stability of the boundary-layer flow. Details on the problem formulation and the governing equations are given by Egorov et al. (2006).

The computational domain is a rectangle $(-0.1 \leqslant x \leqslant 1,0 \leqslant y \leqslant 0.2)$ with its bottom side corresponding to the plate surface in the region $0 \leqslant x \leqslant 1$. The boundary conditions on the plate surface are the no-slip condition $(u, v)=0$ and the adiabatic wall condition $\partial T_{w} / \partial y=0$ for the steady-state solution. On the bottom side in the region $-0.1 \leqslant x<0$, the symmetry conditions are imposed. On the outflow boundary, the unknown dependent variables are extrapolated using the linear approximation. On the inflow and upper boundaries, the conditions correspond to an undisturbed free stream. The upper boundary is located above the induced shock wave.

The problem is solved numerically using the implicit second-order finite-volume method described by Egorov et al. (2006). Two-dimensional Navier-Stokes equations are approximated by a shock-capturing scheme. This is of particular importance for simulation of receptivity processes in which free-stream disturbances pass through the shock and induce its oscillations. The shock-capturing scheme allows us to simulate the disturbance dynamics in the leading-edge vicinity, where receptivity to free-stream disturbances is most pronounced. The advection terms are approximated by the third-order WENO scheme (Jiang \& Shu 1996). Although this computational scheme is dissipative, its numerical dissipation can be suppressed using a sufficiently fine computational grid. Herein the grid has $2201 \times 301$ nodes. There are at least 45 grid nodes per the disturbance wavelength and approximately 400 time steps per the disturbance period. The grid is clustered in the direction normal to the plate surface so that the boundary-layer region contains approximately $50 \%$ of nodes. As shown by Egorov et al. (2006, 2008), the foregoing grid resolution is appropriate for simulations of the boundary-layer stability and receptivity. The governing equations, the code 


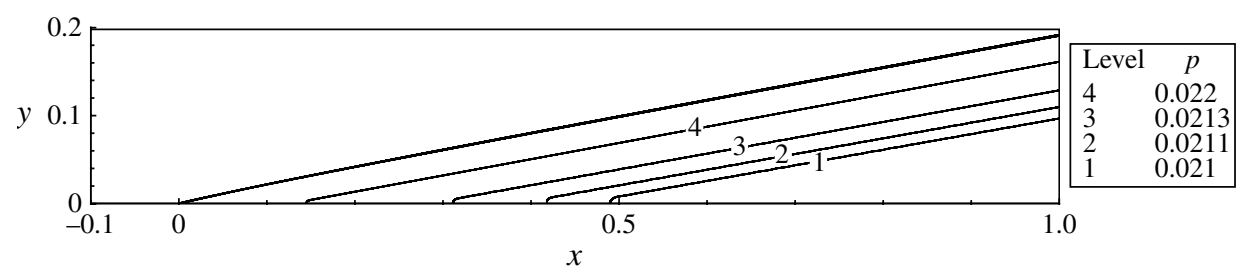

FIgURE 1. The mean-flow pressure field over a flat plate at $M_{\infty}=6$ and $R e_{L}=2 \times 10^{6}$.

algorithm as well as its implementations and validations can be found in Egorov et al. (2006).

First, a steady-state solution without the introduced disturbances is calculated to provide the mean flow. The steady-state pressure field shown in figure 1 indicates that the viscous-inviscid interaction between the boundary layer and the free stream leads to a shock wave emanating from the plate leading edge. As will be shown later, this shock plays a crucial role in the receptivity to the free-stream temperature spottiness.

Second, unsteady disturbances are introduced into the flow for simulations of the receptivity process. The temperature disturbance $T_{0}^{\prime}(x, y ; t)$ is imposed on the steadystate field in the station $x=x_{0}$ as

$$
\begin{aligned}
& T_{0}^{\prime}(x, y ; t)=\varepsilon \delta\left(x-x_{0}\right) \exp \left[-\left(y-y_{0}\right)^{2} / \sigma_{0}^{2}\right] \sin \omega t, \quad t>0, \\
& \delta\left(x-x_{0}\right)=\left\{\begin{array}{ll}
1, & x=x_{0} \\
0, & x \neq x_{0}
\end{array}\right\}
\end{aligned}
$$

where $\varepsilon$ is a small parameter representing the disturbance amplitude and $\omega=\omega^{*} L^{*} / U_{\infty}^{*}$ is the non-dimensional circular frequency. The Gaussian distribution versus $y$ is centred at the point $y_{0}$ and has the characteristic width $\sigma_{0}$.

Note that the temperature spottiness of an actual hypersonic stream essentially depends on many factors and can take quite different shapes. In the inviscid and linear approximation, a temperature disturbance has the form $T^{\prime}=T^{\prime}(x-t, y, z)$ that allows for arbitrary shapes of the temperature spots convected with the free-stream speed. The particular condition (2.1) was chosen because it gives a well-determined row of equally spaced versus $x$ spots that helps to perform parametric studies and identify basic trends of receptivity.

The temperature spottiness has amplitude $\varepsilon=0.1$ at which the receptivity process is predominantly linear. This was confirmed by preliminary computations at various values of $\varepsilon$. The disturbance frequency $\omega=260$ corresponds to the frequency parameter $\mathrm{F}=\omega / \operatorname{Re}=1.3 \times 10^{-4}$ at which the Mack second mode is unstable for $x>0.7$. The temperature disturbance is zero on the plate surface, $T_{w}^{\prime}=0$. The different initial locations $x_{0}, y_{0}$ of the spot centre and different spot sizes $\sigma_{0}$ are considered. In each case, the computations are carried out until a quasi-periodic regime is reached.

The numerical accuracy of the computational results was evaluated by the grid refinement studies. The grid was doubled alternately in the streamwise and wallnormal direction. The first refined grid has $4401 \times 301$ nodes, while the second has $2201 \times 601$ nodes. Numerical simulation of receptivity process is repeated using these grids. Comparisons of the wall-pressure disturbance amplitude shows that the maximal difference is less than $10 \%$ with the streamwise refinement (the amplitude is higher than in the baseline grid case) and it is $5.2 \%$ with the wall-normal refinement (the amplitude is lower than in the baseline grid case). Because the baseline grid captures 


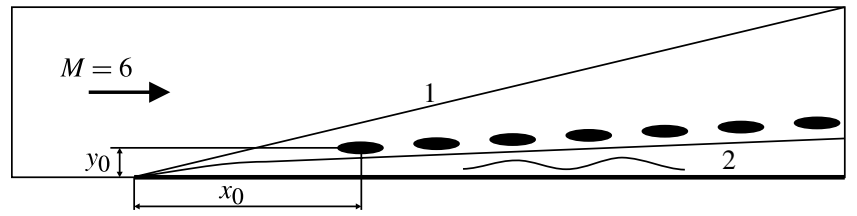

FIGURE 2. Scheme of receptivity to temperature spots propagating over the boundary layer: 1 , shock; 2, boundary-layer disturbances.

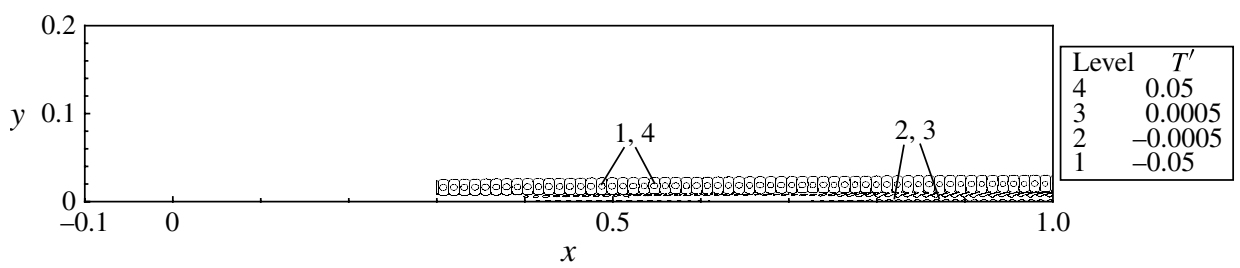

FIgURE 3. Temperature disturbance field for the case of $y_{0}=2 \delta_{e}$ and $\sigma_{0}=0.5 \delta_{e}$.

all trends and structures of the boundary-layer disturbances with sufficiently high accuracy, it was used for the numerical simulations reported hereafter.

\section{Receptivity to temperature disturbances induced downstream of the shock}

\subsection{Numerical results}

First, we consider cases where the temperature spots are induced at the initial station $x_{0}=0.3$ located downstream of the bow shock. This allows us to avoid the spot-shock interaction, which leads to the excitation of acoustic waves and vortical disturbances. These effects are considered later. The temperature spottiness evolution is schematically shown in figure 2 . The spots are induced above the upper boundary-layer edge and propagate downstream with the mean flow speed. Therefore, the temperature disturbance has approximately sinusoidal distribution versus $x$ (with neglecting the boundary-layer growth).

Figure 3 shows the temperature disturbance field in the case of $y_{0}=2 \delta_{e}$ and $\sigma_{0}=0.5 \delta_{e}$. Hereafter $\delta_{e}=0.008$ is the boundary-layer thickness in the mid station $x=0.5$. The spots follow the inviscid flow streamlines, which are slightly displaced by the boundary layer. The disturbance amplitude slowly decreases due to viscous dissipation while the spot size remains practically constant. A similar behaviour of the disturbance field is observed in the cases of $y_{0}=\delta_{e}, 1.25 \delta_{e}, 1.5 \delta_{e}, 1.75 \delta_{e}, 2 \delta_{e}$ and $\sigma_{0}=0.125 \delta_{e}, 0.25 \delta_{e}, 0.375 \delta_{e}, 0.5 \delta_{e}$.

It is convenient to interpret the disturbance evolution using the phase speed distributions $c_{x}(x)$ of different normal modes. In figure 4 , the curves ' $F$ ' and ' $S$ ' show the phase speeds of modes $\mathrm{F}$ and $\mathrm{S}$, respectively, predicted by the LST. This terminology was proposed by Fedorov (2003) in connection with asymptotic behaviour of these modes near the plate leading edge. A detailed discussion of the terminology issue is given by Fedorov \& Tumin (2010). As $x$ tends to zero, the phase speed of mode $\mathrm{F}$ tends to $c_{x}=1+1 / M_{\infty}$ of the free-stream fast acoustic wave (curve ' $\mathrm{F}$ '), whereas the phase speed of mode $\mathrm{S}$ tends to $c_{x}=1-1 / M_{\infty}$ of the free-stream slow acoustic wave (curve ' $S$ '). Note that LST results shown in figure 4 were 


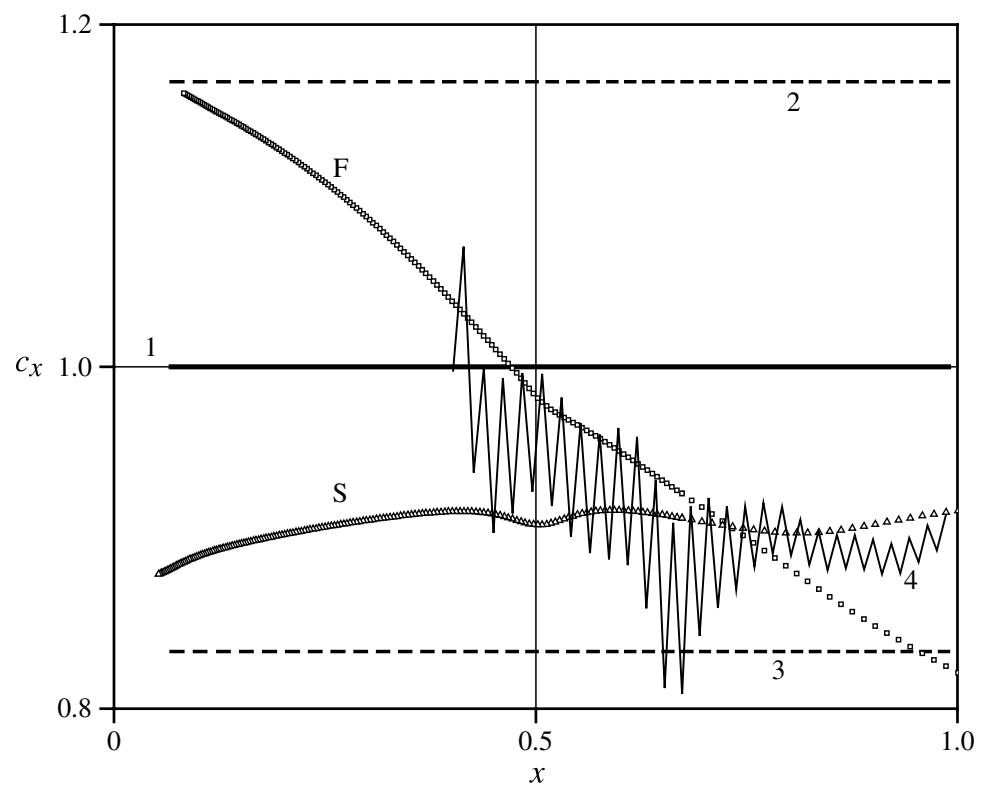

FIGURE 4. The phase speed distributions versus $x$ at $y_{0}=\delta_{e}$ and $\sigma_{0}=0.125 \delta_{e}: 1$, free-stream entropy wave; 2 , free-stream fast acoustic; 3 , free-stream slow acoustic wave; $\mathrm{F}$, mode $\mathrm{F}$ predicted by LST; S, mode S predicted by LST; 4, disturbances induced in the boundary layer by the temperature spottiness (DNS).

obtained using the Navier-Stokes mean-flow solution. The phase speed distributions predicted by DNS are approximately calculated using the wall-pressure disturbance $p_{w}^{\prime}(x)$ as $c_{x}(x) \approx \omega \Delta x / \pi$, where $\Delta x$ is the distance between neighbouring zeros of the function $p_{w}^{\prime}(x)$. The curve 4 shows the phase speed for the disturbances induced in the boundary layer by the temperature spottiness having $y_{0}=\delta_{e}$ and $\sigma_{0}=0.125 \delta_{e}$. The DNS results are qualitatively the same for the other cases and they correlate well with the LST predictions.

Namely, the temperature disturbances propagate downstream with the phase speed $c_{x} \approx 1$ approximately equal to the flow speed (line 1 in figure 4 ), and weakly interact with the boundary-layer flow. Then, approximately at $x=0.5$, they are synchronized with mode $F$ (curve ' $F$ ' crosses line 1) and excite the latter. The pressure disturbances of mode $\mathrm{F}$ have a one-cell structure in the boundary layer (figure $5 a, 0.45 \leqslant x \leqslant 0.55$ ). The corresponding temperature field is shown in figure $5(b)$. Because mode $\mathrm{F}$ is stable, its amplitude decreases downstream from the synchronization point $x \approx 0.5$. This is clearly seen in the wall-pressure disturbance distribution shown in figure 6 . Further downstream, mode $\mathrm{F}$ is synchronized with mode $\mathrm{S}$ and excites the latter via the inter-modal exchange mechanism described by Fedorov \& Khokhlov (2001). This occurs just upstream from the neutral point $x \approx 0.7$. The inter-modal exchange takes place in a local region where the eigenvalues of modes $\mathrm{F}$ and $\mathrm{S}$ are close to each other. In this region located near the branch point of the discrete spectrum, the non-parallel effect leads to switching from one mode to the other. By solving the local problem and matching its solution with the outer solutions, Fedorov \& Khokhlov (2001) derived a rule which couples the amplitudes of incoming and outgoing modes. In accord with this rule, in the leading-order approximation the amplitude of the outgoing mode $\mathrm{S}$ 

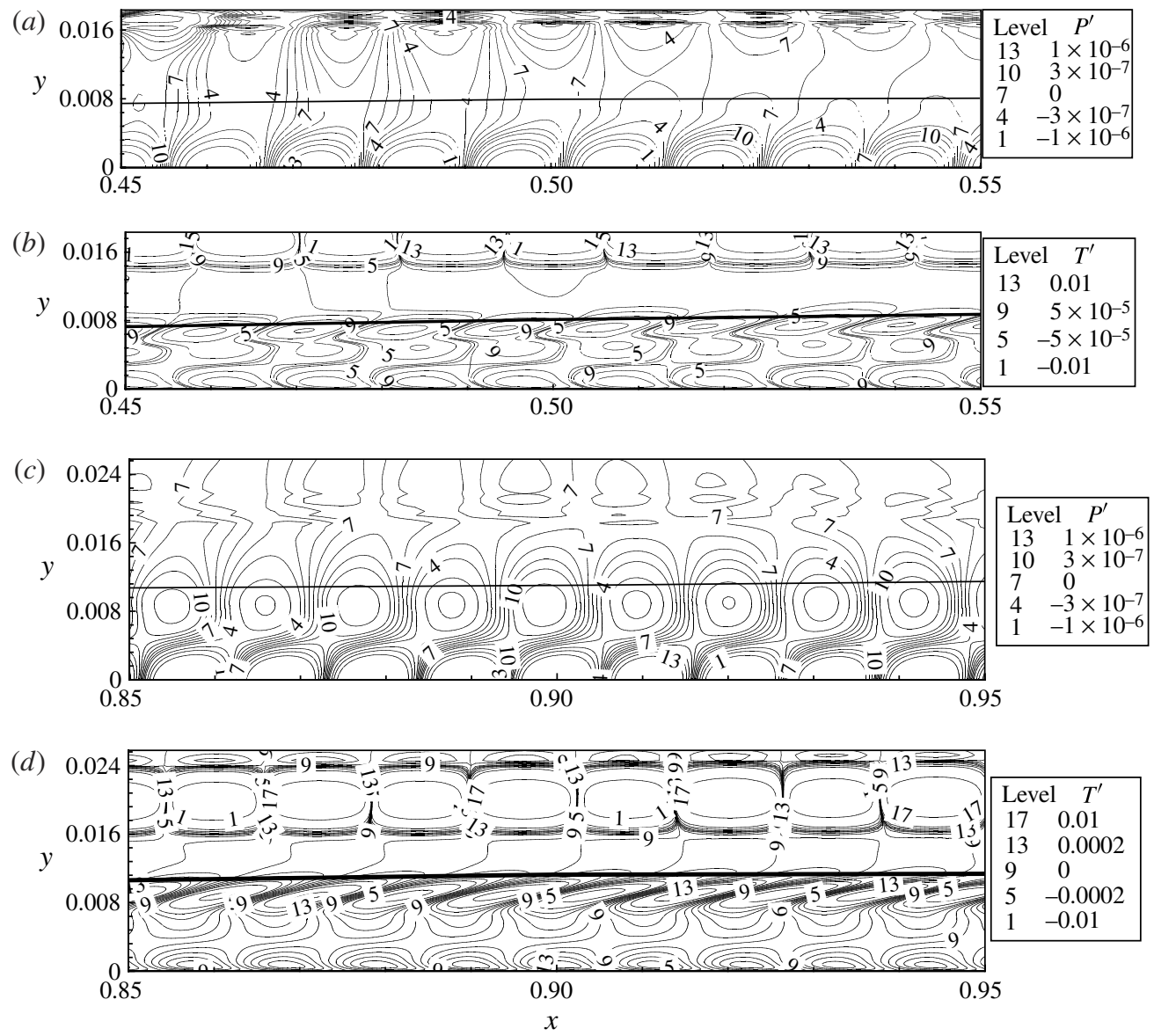

Figure 5. (a) Pressure disturbance field for $y_{0}=2 \delta_{e}, \sigma_{0}=0.125 \delta_{e}, 0.45 \leqslant x \leqslant 0.55$, $0 \leqslant y \leqslant 0.018$, thick line corresponds to the boundary-layer thickness. (b) Temperature disturbance field for $y_{0}=2 \delta_{e}, \sigma_{0}=0.125 \delta_{e}, 0.45 \leqslant x \leqslant 0.55,0 \leqslant y \leqslant 0.018$. (c) Pressure disturbance field for $y_{0}=2 \delta_{e}, \sigma_{0}=0.125 \delta_{e}, 0.85 \leqslant x \leqslant 0.95,0 \leqslant y \leqslant 0.018$. (d) Temperature disturbance field for $y_{0}=2 \delta_{e}, \sigma_{0}=0.125 \delta_{e}, 0.85 \leqslant x \leqslant 0.95,0 \leqslant y \leqslant 0.018$.

equals to the amplitude of the incoming mode F. Ultimately, the unstable mode $\mathrm{S}$ amplifies downstream as shown in figure $6(a)$ and forms two-cell structures in the pressure field (figure $5 c, 0.85 \leqslant x \leqslant 0.95$ ). The corresponding temperature field is shown in figure $5(d)$.

The wall-pressure disturbances for different $y_{0}$ and $\sigma_{0}$ (figure $6 a-c$ ) indicate that there are two local maxima of the disturbance amplitude. The first maximum is observed at $x \approx 0.5$ and corresponds to the synchronization between mode $\mathrm{F}$ and the entropy mode of continuous spectrum. The second maximum is located at $x \approx 0.9$ and associated with mode $\mathrm{S}$.

In the cases of relatively 'thick' spottiness propagating near the upper boundarylayer edge $\left(y_{0}=\delta_{e}\right)$, there is no local maximum at $x \approx 0.5$. These spots are partially immersed into the boundary layer (see figure 7a,b) that affects their evolution and, hence, the receptivity process. 

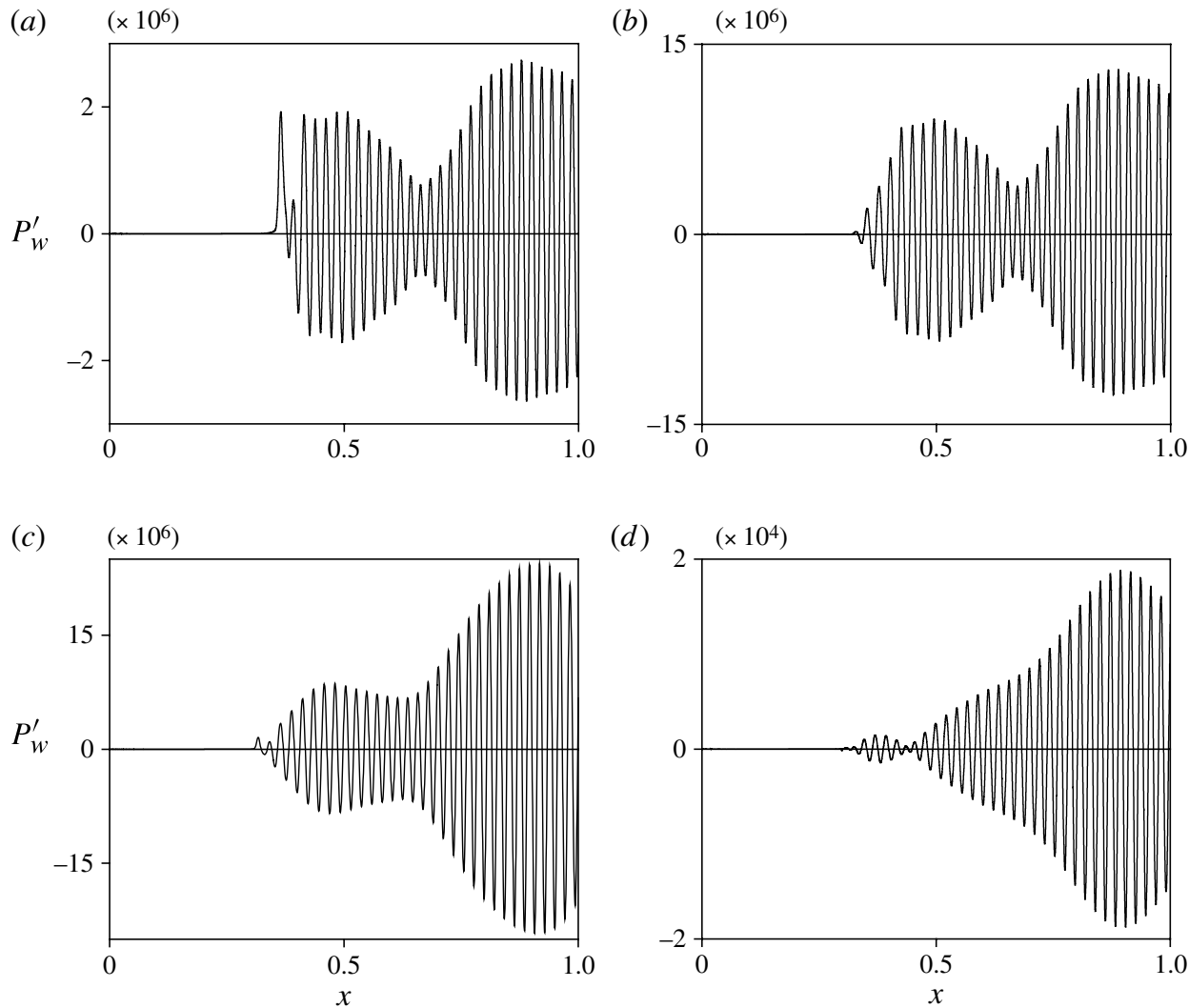

FIGURE 6. (a) Wall-pressure disturbance distribution for $y_{0}=2 \delta_{e}, \sigma_{0}=0.125 \delta_{e}$. (b) Wallpressure disturbance distribution for $y_{0}=2 \delta_{e}, \sigma_{0}=0.5 \delta_{e}$. (c) Wall-pressure disturbance distribution for $y_{0}=\delta_{e}, \sigma_{0}=0.125 \delta_{e}$. $(d)$ Wall-pressure disturbance distribution for $y_{0}=\delta_{e}$, $\sigma_{0}=0.5 \delta_{e}$.
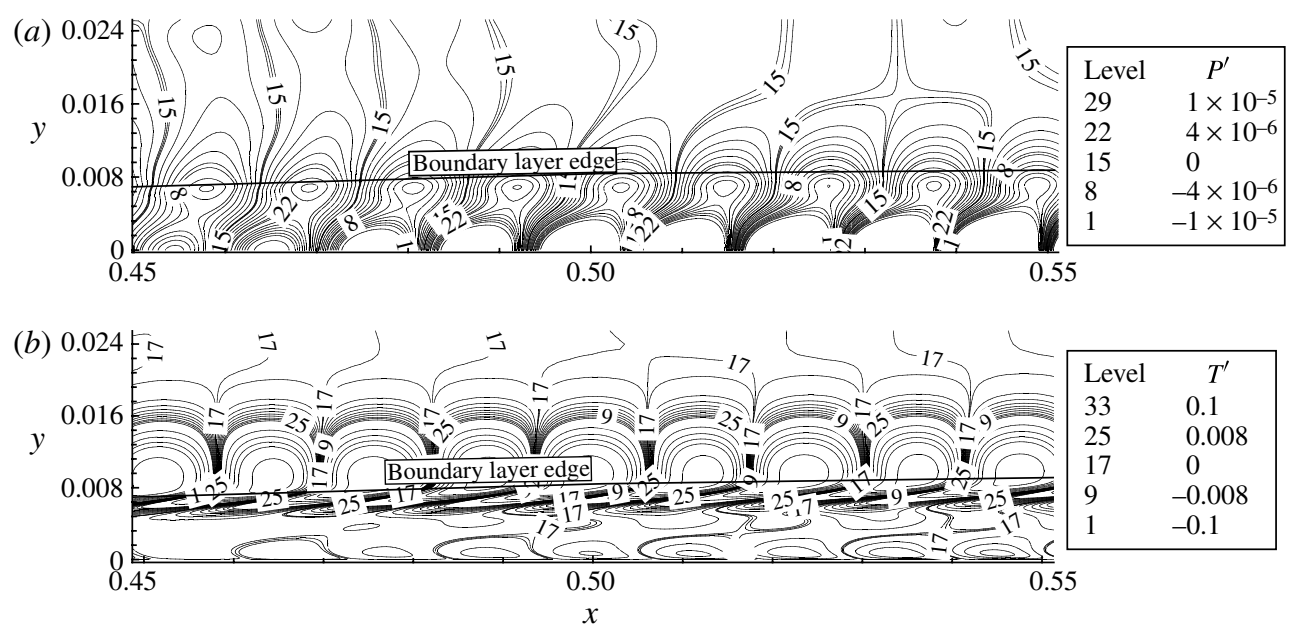

Figure 7. (a) Pressure disturbance field for $y_{0}=\delta_{e}, \sigma_{0}=0.5 \delta_{e}, 0.45 \leqslant x \leqslant 0.55$. (b) Temperature disturbance field for $y_{0}=\delta_{e}, \sigma_{0}=0.5 \delta_{e}, 0.45 \leqslant x \leqslant 0.55$. 

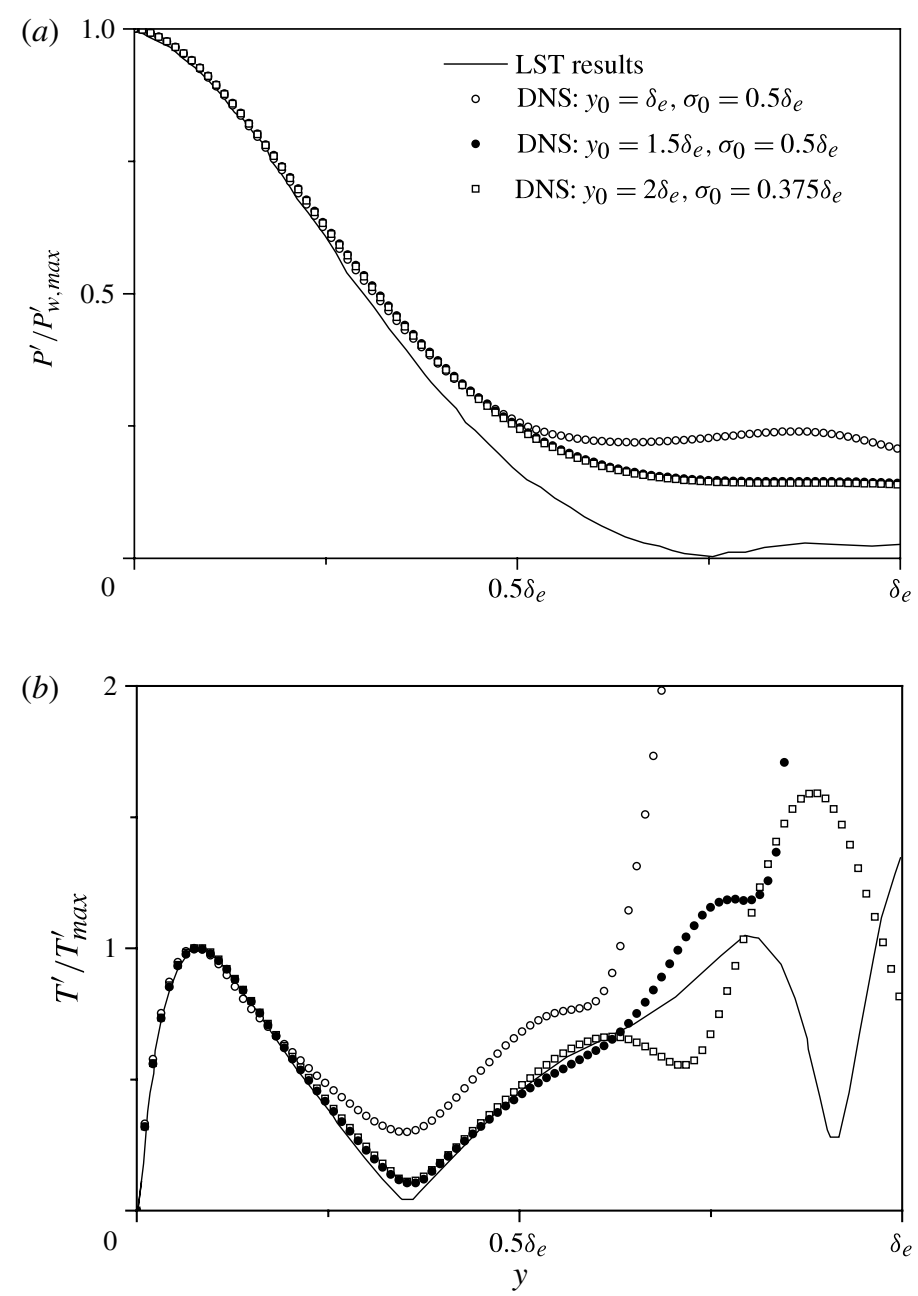

FIGURE 8. (a) Pressure disturbance eigenfunctions in the boundary layer at $x=0.5$. (b)

Temperature disturbance eigenfunctions in the boundary layer at $x=0.5$.

In figures $8(a)$ and $8(b)$, the pressure and temperature disturbances resulted from DNSs are compared with the corresponding eigenfunctions of mode $F$ predicted by LST at $x=0.5$. Here the pressure profiles are normalized by the wall-pressure disturbance amplitude $p_{w}^{\prime}$ at $x=0.5$ while the temperature profiles are normalized by the temperature disturbance amplitude in the first maximum of its $y$-distribution at $x=0.5$. The temperature distributions are close to each other in the near-wall region $y<0.005$. For $y>0.005$, they are essentially different because the DNS solution is affected by the temperature spottiness. Discrepancies between the pressure profiles in the outer region $y>0.004$ indicate that the spots generate relatively weak acoustic waves, which are not present in the LST solution.

\subsection{Theoretical assessments}

For a first-cut modelling of the receptivity mechanism, the mean flow is approximated by the self-similar compressible Blasius solution with the upper boundary-layer edge 


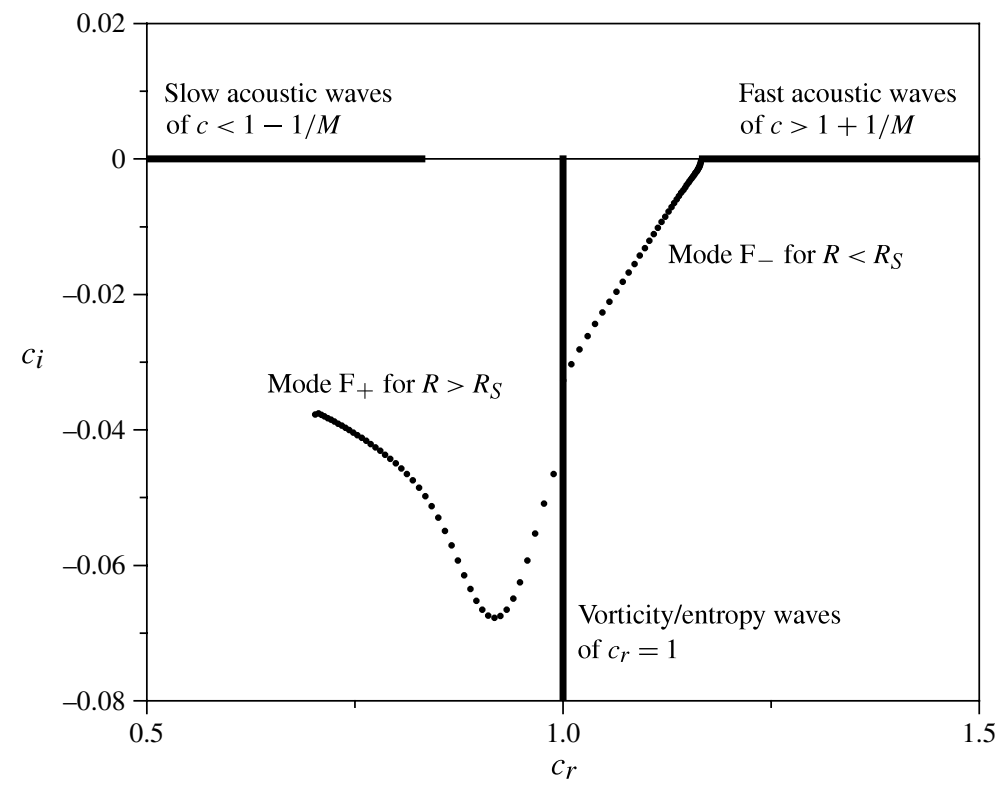

FIGURE 9. Trajectory of the mode F pole $\alpha_{F}(R, \omega)$ (dots) in the complex $c$-plane, thick black lines show branches of the continuous spectrum.

parameters (denoted by subscript ' $e$ ') taken from DNSs. The temperature spot shape is written in the similarity variables $(R, \eta)=\left(\sqrt{U_{e}^{*} x^{*} / \nu_{e}^{*}}, y^{*} / \sqrt{x^{*} \nu_{e}^{*} / U_{e}^{*}}\right)$ as

$$
T^{\prime}(\eta)=\varepsilon \exp \left[-\frac{\left(\eta-\eta_{0}\right)^{2}}{\sigma_{0}^{2}}\right]=\varepsilon f(\eta),
$$

where $\eta_{0}(R)$ is the vertical coordinate of the spot centre and $v_{e}^{*}$ is the kinematic viscosity coefficient. It is assumed that the spot size is of the order of the boundarylayer thickness, $\sigma_{0}=O(1)$. The disturbance vector is introduced as $\boldsymbol{\Psi}(R, \eta, t)=$ $\left(u^{\prime}, \partial u^{\prime} / \partial \eta, v^{\prime}, p^{\prime}, T^{\prime}, \partial T^{\prime} / \partial \eta\right)^{\mathrm{T}}$, and the temperature spot disturbance is expressed in the form

$$
\boldsymbol{\Psi}(R, \eta, t)=\varepsilon \boldsymbol{A}_{T}(\eta, R) \exp \left[2 \mathrm{i} \int_{R_{S}}^{R} \alpha_{T}(R) \mathrm{d} R-\mathrm{i} \omega t\right],
$$

where $\boldsymbol{A}_{T}=(0,0,0,0, f, \mathrm{~d} f / \mathrm{d} \eta)^{\mathrm{T}}, \alpha_{T}$ is a wave number and $R_{S}$ corresponds to the initial station $x_{S}$.

As previously shown, mode $\mathrm{F}$ is synchronized with vorticity/entropy waves at $x_{S} \approx 0.5$ corresponding to $R_{S} \approx 1000$. This is illustrated by figure 9 , where the trajectory of eigenvalue $\alpha_{F}(R, \omega)$ is shown in the complex $c$-plane. The pole $\alpha_{F}(R, \omega)$ coalesces with the vorticity/entropy branch of continuous spectrum as the Reynolds number approaches the synchronization point $R \rightarrow R_{S}-0$. For $R>R_{S}$, this pole goes to the lower sheet of the Riemann surface. Another pole is observed in the downstream region $R>R_{S}$. It coalesces with the vorticity/entropy branch as $R \rightarrow R_{S}+0$, and goes to the upper sheet of the Riemann surface for $R<R_{S}$. Because one pole does not convert into the other, we should distinguish the modes associated with these poles. Hereafter, the mode observed for $R<R_{S}$ (or $R>R_{S}$ ) is called mode $\mathrm{F}_{-}$(or $\mathrm{F}_{+}$). The mode $\mathrm{F}_{+}$is synchronized with mode $\mathrm{S}$ somewhere downstream from the station $R_{S}$. 
It is assumed that the temperature spots, which are localized outside the boundary layer, smoothly evolve through the point $R_{S}$ and excite mode $\mathrm{F}_{+}$in the region $R>R_{S}$. Hence, a problem describing excitation of mode $\mathrm{F}_{+}$can be formulated for $R>R_{S}$. Consider a partial solution consisting of mode $\mathrm{F}_{+}$(with the eigenfunction $\boldsymbol{A}_{F}$ and eigenvalue $\alpha_{F}$ ) and the temperature-spot disturbance (with the shape function $\boldsymbol{A}_{T}$ and wavenumber $\alpha_{T}$ )

$$
\begin{aligned}
\Psi= & C_{F}(R) \boldsymbol{A}_{F}(R, \eta) \exp \left[2 \mathrm{i} \int_{R_{S}}^{R} \alpha_{F}(R) \mathrm{d} R-\mathrm{i} \omega t\right] \\
& +\varepsilon \boldsymbol{A}_{T}(\eta, R) \exp \left[2 \mathrm{i} \int_{R_{S}}^{R} \alpha_{T}(R) \mathrm{d} R-\mathrm{i} \omega t\right] .
\end{aligned}
$$

In accord with the biorthogonal eigenfunction decomposition method (Zhigulev \& Tumin 1987; Gaydos \& Tumin 2004), the weight coefficient $C_{F}$ is governed by the problem

$$
\begin{aligned}
\frac{\mathrm{d} C_{F}}{\mathrm{~d} R} & =\varepsilon W_{T, F}(R) \exp \left[2 \mathrm{i} \int_{R_{S}}^{R}\left[\alpha_{T}(R)-\alpha_{F}(R)\right] \mathrm{d} R\right], \\
R=R_{S}+0: C_{F} & =\varepsilon \frac{\left\langle H_{2} \boldsymbol{A}_{T}, \boldsymbol{B}_{F}\right\rangle}{\left\langle H_{2} \boldsymbol{A}_{F}, \boldsymbol{B}_{F}\right\rangle}, \\
W_{T, F}(R) & =-\frac{1}{R} \frac{\left\langle H_{2}\left(\frac{1}{2} R \frac{\partial \boldsymbol{A}_{T}}{\partial R}-\frac{\eta}{2} \frac{\partial \boldsymbol{A}_{T}}{\partial \eta}\right), \boldsymbol{B}_{F}\right\rangle+\left\langle H_{3} \boldsymbol{A}_{T}, \boldsymbol{B}_{F}\right\rangle}{\left\langle H_{2} \boldsymbol{A}_{F}, \boldsymbol{B}_{F}\right\rangle} .
\end{aligned}
$$

Here the scalar product is defined as $\langle\boldsymbol{F}, \boldsymbol{G}\rangle=\int_{0}^{\infty}(\boldsymbol{F}, \boldsymbol{G}) \mathrm{d} \eta$, non-zero elements of the matrix $\mathrm{H}_{2}$ and $\mathrm{H}_{3}$ are given by Gaydos \& Tumin (2004), $\boldsymbol{B}_{F}$ is eigenfunction of the adjoined problem. The initial condition (3.5) is obtained by expansion of the temperature-spot shape function $\boldsymbol{A}_{T}$ into the biorthogonal eigenfunction system $\{\boldsymbol{A}, \boldsymbol{B}\}$ at $R=R_{S}+0$. In the derivation of the amplitude equation (3.4), it is assumed that the temperature spot is weakly affected by the excited boundary-layer modes, i.e. the nonlinear effects are neglected. A formal solution of the problem (3.4)-(3.6) is expressed as

$$
\begin{aligned}
R>R_{S}: C_{F}= & \left.\varepsilon \frac{\left\langle H_{2} \boldsymbol{A}_{T}, \boldsymbol{B}_{F}\right\rangle}{\left\langle H_{2} \boldsymbol{A}_{F}, \boldsymbol{B}_{F}\right\rangle}\right|_{R=R_{S}+0} \\
& +\varepsilon \int_{R_{S}}^{R} W_{T, F}(R) \exp \left[2 \mathrm{i} \int_{R_{S}}^{R}\left[\alpha_{T}(R)-\alpha_{F}(R)\right] \mathrm{d} R\right] \mathrm{d} R .
\end{aligned}
$$

According to (3.6) the coefficient $W_{T, F}$ is associated with non-parallel effects. The first term in the numerator represents streamwise distortions of the spot shape while the second term is related to streamwise distortions of the mean flow. The latter are weak for sufficiently large Reynolds numbers $R$. Assuming that the vertical length scale of the temperature spot is of the order of the boundary-layer thickness, we obtain $W_{T, F}=O\left(R_{S}^{-1}\right)$. Hence, the weight coefficient can be estimated as

$$
C_{F}=\varepsilon\left[\left.\frac{\left\langle H_{2} \boldsymbol{A}_{T}, \boldsymbol{B}_{F}\right\rangle}{\left\langle H_{2} \boldsymbol{A}_{F}, \boldsymbol{B}_{F}\right\rangle}\right|_{R=R_{S}+0}+O\left(R_{S}^{-1}\right)\right] .
$$


Thus, the amplitude of mode $\mathrm{F}_{+}$, which is excited in the vicinity of the synchronization point $R_{S}$, can be estimated using the weight coefficient resulted from the expansion of the temperature-spot shape function into the eigenfunctions at $R=R_{S}+0$. An additional excitation in the downstream region $R>R_{S}$ is due to the mean-flow non-parallel effects and can be neglected for large values of $R_{S}$. Note that this approximate model was derived for the cases where the temperature spot is localized outside the boundary layer. Otherwise, the spot distortions induced by the boundary-layer flow cannot be neglected.

\subsection{Receptivity coefficients}

To evaluate the receptivity mechanism and compare the theoretical assessments with the DNS results, we introduce the receptivity coefficients: $(a) K_{T}=T_{\max 1}^{\prime} / \varepsilon$, where $T_{\max 1}^{\prime}$ is the temperature disturbance amplitude measured in the first maximum of its $y$-distribution at $x=0.5$ (see figure $8 b$ ); (b) $K_{P}=p_{w}^{\prime} \gamma M_{e}^{2} / \varepsilon$, where $p_{w}^{\prime}$ is the pressure disturbance amplitude at $y=0$ and $x=0.5, M_{e}$ is the Mach number at the upper boundary-layer edge.

Note that the spot parameters $y_{0}, \sigma_{0}$ at $x=0.5$ are slightly different from their values at $x=0.3$ because of the boundary-layer displacement effect and viscous dissipation. For this reason, the theoretical receptivity coefficients are calculated using the spot parameters taken from the DNS solution at $x_{S}=0.5$. The computations were performed for the cases:

(a) spots of $\sigma_{0}=0.125 \delta_{e}$ at various distances from the wall $\delta_{e} \leqslant y_{0} \leqslant 2 \delta_{e}$;

(b) spots of $\sigma_{0}=0.5 \delta_{e}$ at various distances from the wall $\delta_{e} \leqslant y_{0} \leqslant 2 \delta_{e}$;

(c) spots of various sizes $0.125 \delta_{e} \leqslant \sigma_{0} \leqslant 0.5 \delta_{e}$ at the fixed distance from the wall $y_{0}=2 \delta_{e}$.

The receptivity coefficients for cases 1 and 2 are shown in figure $10(a)$ and $(b)$, respectively. As expected, the agreement between DNS and theory improves as $y_{0}$ increases. For relatively small $y_{0}$, the spot shape is affected by the boundary-layer flow that is not taken into account by the theoretical model. This effect is stronger for spots of larger size $\sigma_{0}$. Figure 11 shows the difference between Gaussian distributions (solid lines) and DNS solutions (dots) at $x=0.5$ for $y_{0}=\delta_{e}, \sigma_{0}=0.5 \delta_{e}$ and $y_{0}=1.75 \delta_{e}$, $\sigma_{0}=0.5 \delta_{e}$. The near-wall portion of the spot is suppressed owing to the boundarylayer displacement effect, while the upper part of the spot (located far off the boundary layer) is not affected. An oscillatory behaviour of $K\left(y_{0}\right)$ in figure $10(a)$ is due to the fact that the spot size is of the order of the length scale related to oscillations of the eigenfunction $\boldsymbol{B}_{F}$ outside the boundary layer. This causes oscillations of the scalar product $\left\langle\mathrm{H}_{2} \boldsymbol{A}_{T}, \boldsymbol{B}_{F}\right\rangle$ from (3.8). For relatively 'thick' spots, the oscillations are averaged out and the distribution $K\left(y_{0}\right)$ is smooth (figure $10 b$ ).

The receptivity coefficients for case 3 are shown in figure 12 . The theoretical distribution of $K\left(\sigma_{0}\right)$ agrees well with DNS. In this case, the spots are located sufficiently far from the boundary-layer edge where the theoretical model works better.

\section{Receptivity to free-stream spots passing through the bow shock}

In actual conditions the free-stream temperature disturbances pass through the bow shock as schematically shown in figure 13. Herein, effects associated with the spot-shock interaction are simulated numerically. Computations are carried out for the spots of $\sigma_{0}=0.125 \delta_{e}$. The initial vertical locus $y_{0}$ varies from 0 to $2 \delta_{e}$, while the streamwise locus is $x_{0}=-0.1$. As an example, figure 14(a) and (b) show the 

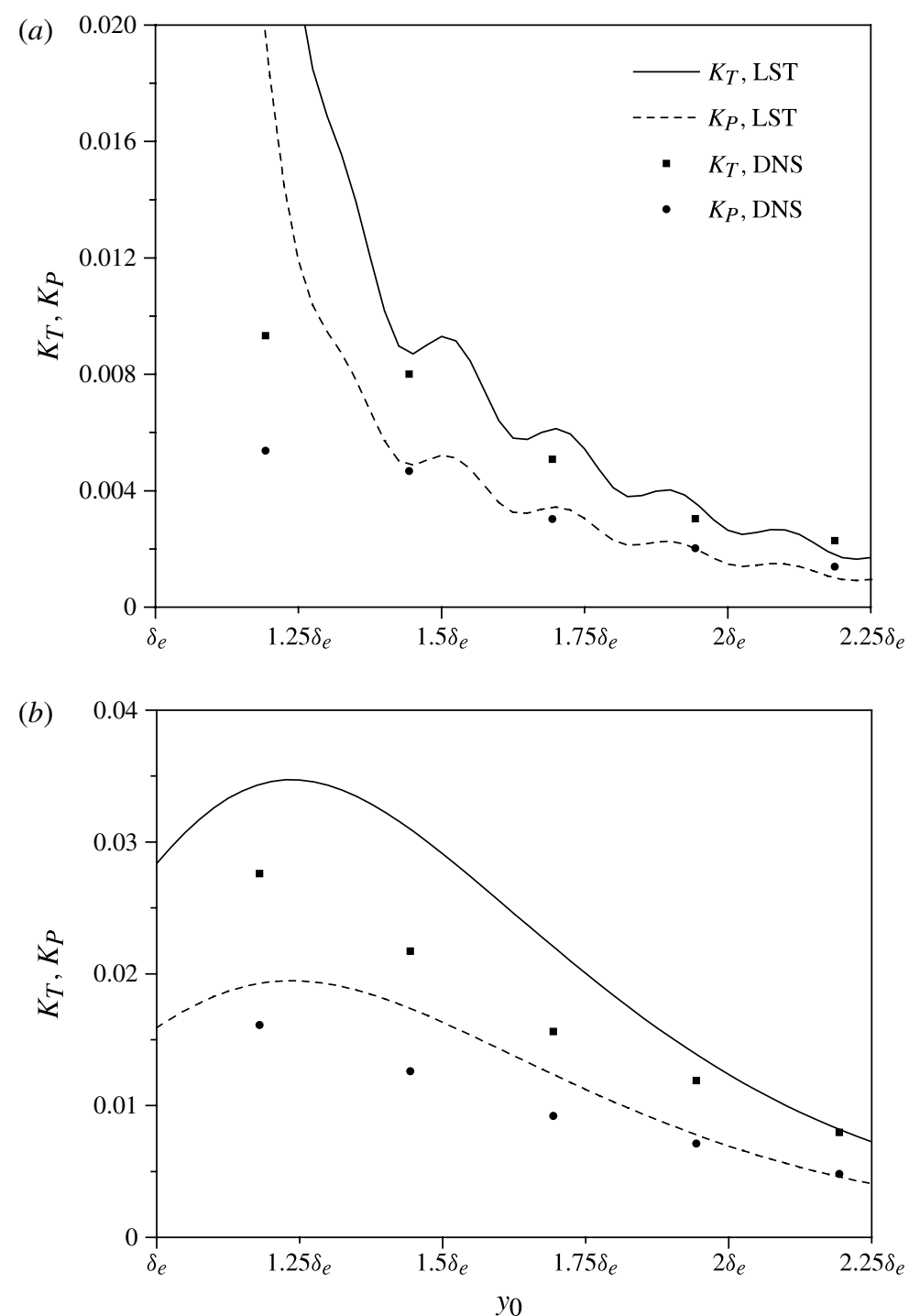

FIGURE 10. (a) Receptivity coefficients versus $y_{0}$ at $x=0.5, \sigma_{0}=0.125 \delta_{e}$. (b) Receptivity coefficients versus $y_{0}$ at $x=0.5, \sigma_{0}=0.5 \delta_{e}$.

disturbance fields for the pressure and temperature, respectively, in the case of $y_{0}=2 \delta_{e}$. In accordance with the linear theory, the spots do not generate acoustic disturbances in the uniform stream in front of the shock. Passing through the shock, the spottiness generates slow and fast acoustic waves which propagate downstream and interact with the boundary-layer flow (figure 14a). Because the slow acoustic waves are synchronized with mode $\mathrm{S}$ near the plate leading edge, they can effectively excite the latter (Fedorov 2003). Presumably this mechanism is responsible for the excitation of mode $\mathrm{S}$ (or Mack second mode) which is observed in the DNS solution for $x>0.5$. It should be noted that the spot-shock interaction weakly affects the temperature spots themselves. Their shape and amplitude remain approximately the same behind the shock as shown in figure $14 b$. 


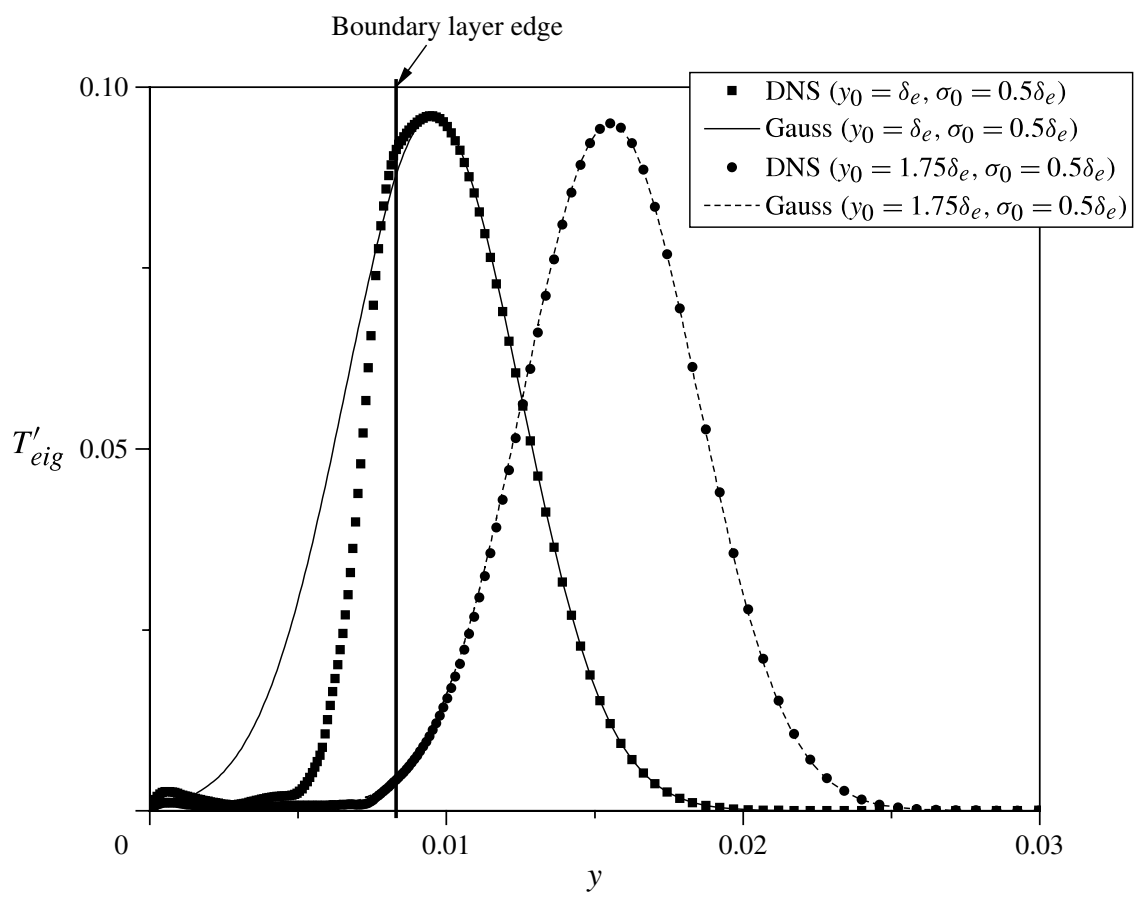

FIGURE 11. The $y$-distributions of the temperature spots at $x=0.5$.

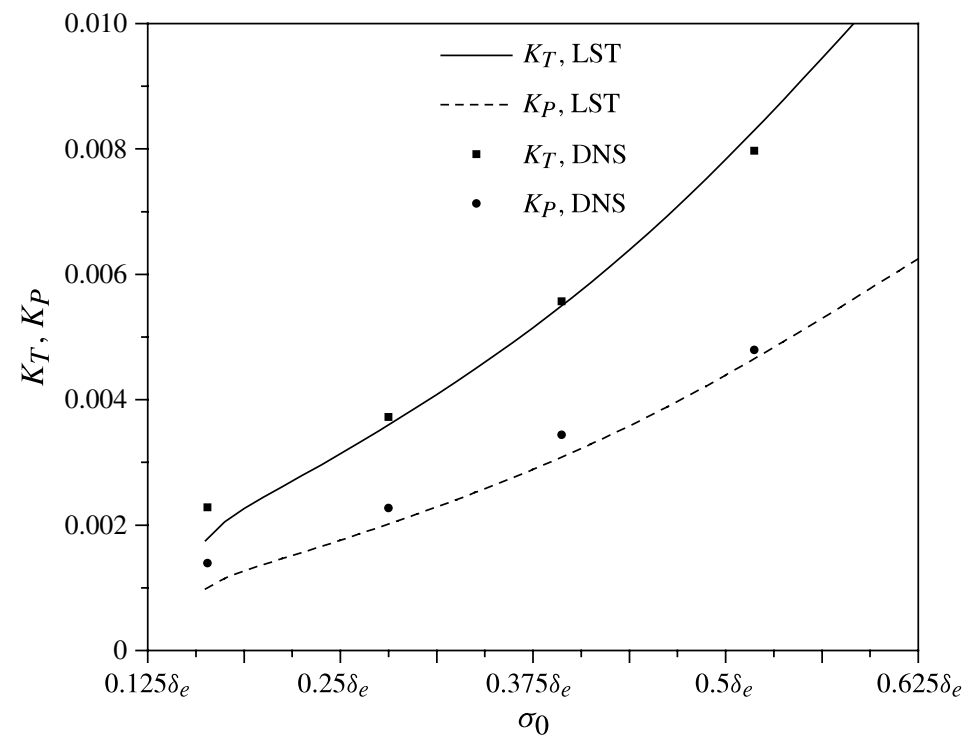

FIGURE 12. Receptivity coefficients versus $\sigma_{0}$ at $x=0.5$ and $y_{0}=2 \delta_{e}$.

The phase speed diagram (figure 15) indicates that mode $S$ is excited upstream from the station $x=0.5$ where mode $\mathrm{F}$ is synchronized with the temperature spots. The wall-pressure disturbances for the case of $y_{0}=2 \delta_{e}, x_{0}=-0.1, \sigma_{0}=0.125 \delta_{e}$ are shown in figure 16. 


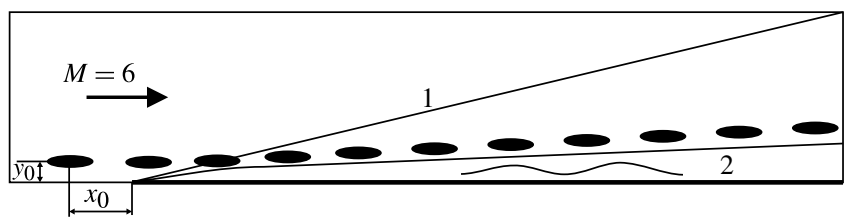

FIGURE 13. Scheme of receptivity to free-stream temperature spots passing through the bow shock: 1, shock; 2, boundary-layer disturbances.
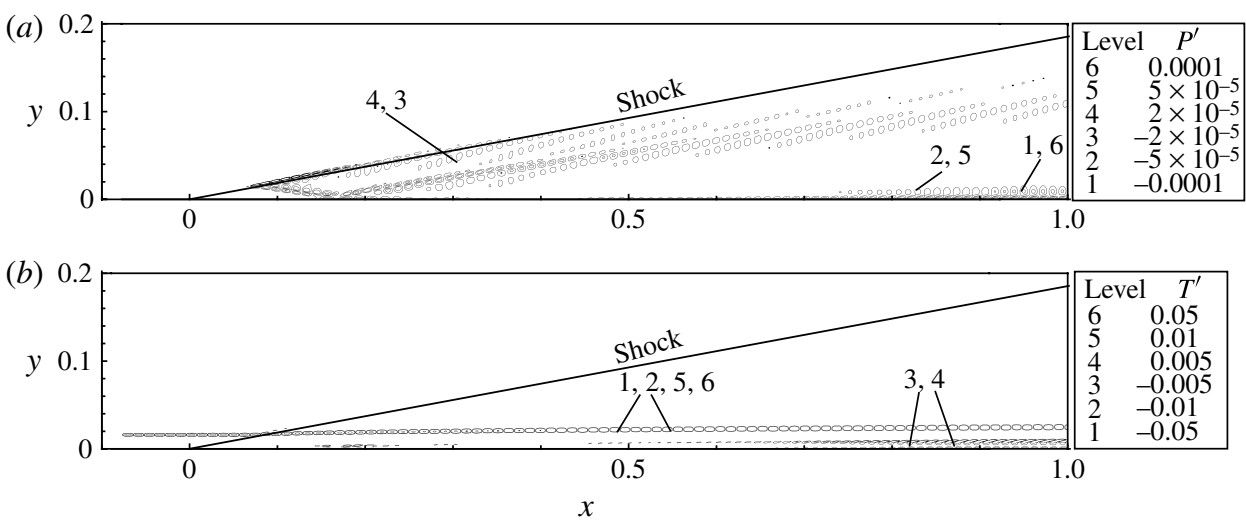

Figure 14. (a) Pressure disturbance field, $y_{0}=2 \delta_{e}, x_{0}=-0.1, \sigma_{0}=0.125 \delta_{e}$. (b) Temperature disturbance field at $y_{0}=2 \delta_{e}, x_{0}=-0.1, \sigma_{0}=0.125 \delta_{e}$.

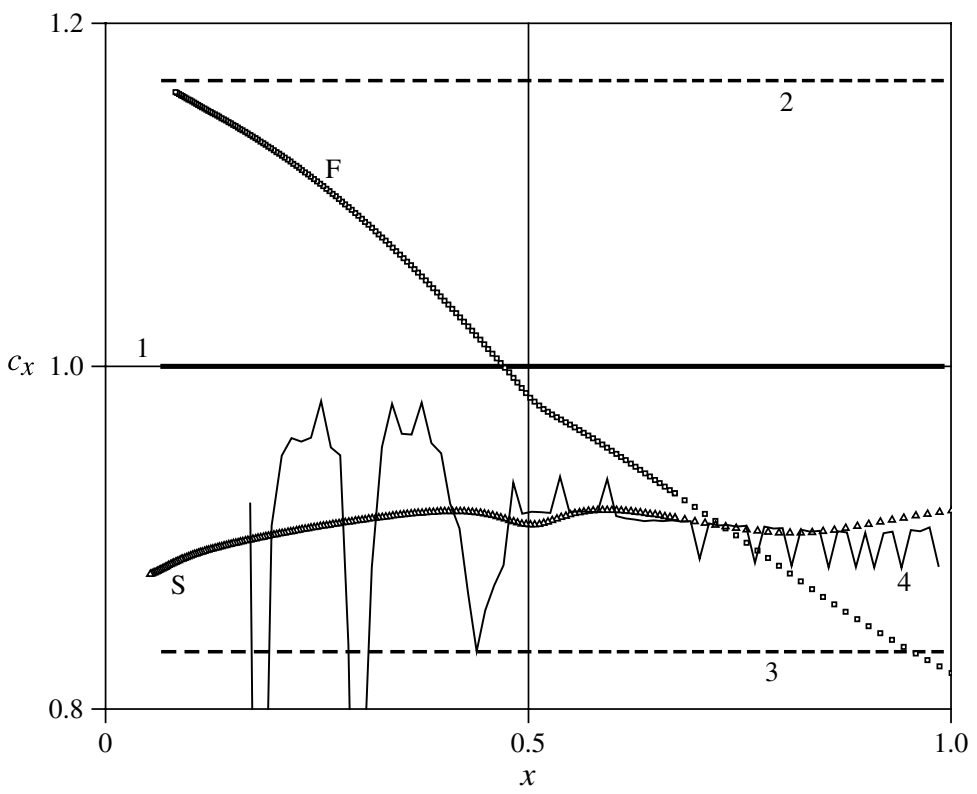

FIGURE 15. The phase speed distribution versus $x$ for $y_{0}=2 \delta_{e}$ and $\sigma_{0}=0.125 \delta_{e}$ : 1 , freestream entropy wave; 2 , free-stream fast acoustic wave; 3 , free-stream slow acoustic wave; F, mode F predicted by LST; S, mode S predicted by LST; 4, disturbances induced in the boundary layer by temperature spottiness (DNS). 


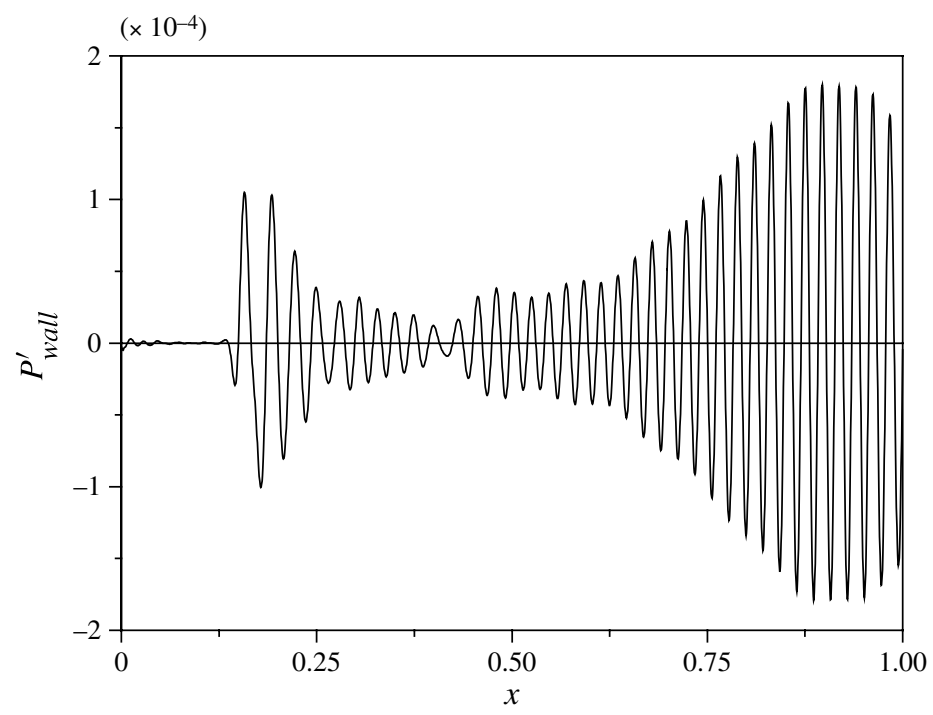

FIGURE 16. The wall-pressure disturbance distribution for $y_{0}=2 \delta_{e}$ and $\sigma_{0}=0.125 \delta_{e}$.
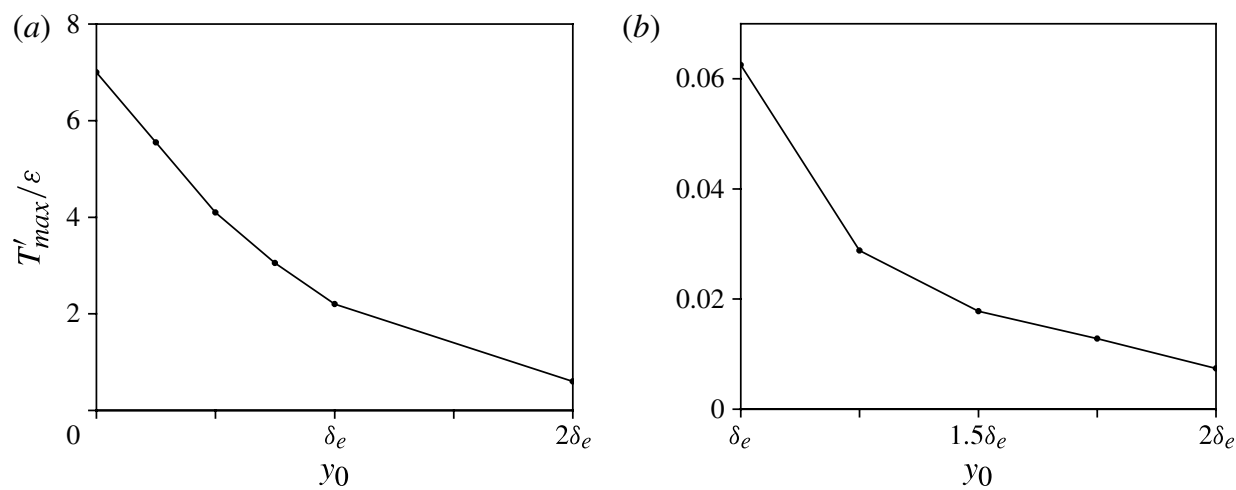

FIGURE 17. (a) Maximal amplitude of the temperature disturbance at $x=0.9$, which is normalized by $\varepsilon$, as a function of $y_{0}$ for $x_{0}=-0.1$. (b) Maximal amplitude of the temperature disturbance at $x=0.9$, which is normalized by $\varepsilon$, as a function of $y_{0}$ for $x_{0}=0.3$.

Thus, the boundary-layer instability is excited by acoustic disturbances rather than by the temperature spots themselves. This conclusion is supported by the data shown in figure $17(a)$ and $(b)$, where the maximum amplitude of temperature disturbance in the boundary layer at $x \approx 0.9$ is plotted as a function of the spottiness vertical locus $y_{0}$ for the case of $x_{0}=-0.1$ (spots are induced in front of the bow shock) and for the case of $x_{0}=0.3$ (spots are induced behind the shock). The spot-shock interaction leads to $\sim 50-100$ times increase of the mode $\mathrm{S}$ amplitude. Because the amplification of instability is the same in the both cases, this difference is associated with receptivity only. As expected the receptivity coefficient decreases as $y_{0}$ increases. In the case of $x_{0}=-0.1$, this trend is due to the weakening of the bow shock and attenuation of cylindrical acoustic waves propagating from the spot-shock interaction 
region to the boundary layer. Moreover, for larger $y_{0}$ the region radiated by the induced acoustic waves is shifted downstream from the leading edge region where receptivity is maximal. In the case of $x_{0}=0.3$, the spot-mode $\mathrm{F}$ interaction decreases with $y_{0}$ because the eigenfunction $\boldsymbol{B}_{F}$ decays exponentially outside the boundary layer.

Thus, the bow shock plays a crucial role in receptivity to free-stream temperature spottiness. The dominant excitation of instability is governed by the two-step mechanism. First, spots interact with the shock and generate acoustic waves. Second, the induced acoustic waves penetrate into the boundary layer and generate unstable waves. Presumably a similar mechanism drives receptivity to free-stream turbulence associated with vortical disturbances.

It should be noted that in the considered herein unit problem the shock is weak. For more practical configurations with blunted leading edges and/or non-zero angles of attack, a much stronger bow shock can significantly enhance the acoustic noise generated by the shock-spot or shock-vortex interaction. The relative role of this noise in the receptivity process essentially depends on the disturbance environment. In conventional wind tunnels it is masked by acoustic waves generated in the turbulent boundary layer on the nozzle and test-section walls. In free flights, where there are no free-stream acoustic perturbations, it may be the major source of disturbances leading to the second-mode dominated transition. However, even in this 'clean' case, there are other competing sources of external forcing such as free-stream particulates and electrostatic discharges (Bushnell 1990). For example, Fedrorov \& Kozlov (2011) showed that particulates of $\sim 10 \mu \mathrm{m}$ size trigger the second-mode wavepackets, which can lead to transition on a sharp wedge flying with hypersonic speeds. In order to identify the dominant receptivity mechanism one should evaluate various sources of external forcing and sort out their receptivity efficiency.

\section{Summary}

DNSs of the receptivity to two-dimensional temperature spottiness were carried out for a flat plate in the free stream at Mach number 6.

In the first series of computations, the temperature spots were induced downstream of the bow shock in order to avoid the spot-shock interaction leading to excitation of acoustic waves and vortical disturbances. These simulations were focused on the receptivity mechanism associated with only one type of external disturbance: entropy waves. Excitation of the boundary-layer modes was predicted for the different sizes and locations of the spots. It was found that mode $\mathrm{F}$ is predominantly generated near the synchronization point, where its phase speed coincides with the phase speed of vorticity/entropy waves of the continuous spectrum. Propagating downstream, mode F is synchronized with unstable mode S (Mack second mode) and excites the latter via the inter-modal exchange mechanism.

An approximate theoretical model describing excitation of mode $\mathrm{F}$ by temperature spots propagating over the boundary-layer upper edge was developed. According to this model the dominant excitation occurs in the vicinity of the synchronization point $x_{S}$ where the mode $\mathrm{F}$ phase speed equals to the phase speed of entropy waves. The mode $\mathrm{F}$ amplitude is estimated by an expansion of the temperature-spot disturbance into the biorthogonal eigenfunction system at $x=x_{S}+0$. The receptivity coefficients (associated with the initial amplitude of mode $\mathrm{F}$ at the synchronization point) were evaluated using the DNSs and the theoretical solutions for different parameters of the temperature spottiness. Comparisons showed that the theory agreed with the DNSs in the cases where the temperature spots were localized outside the boundary layer. 
This theoretical model can be used for rough estimates of the initial amplitudes of mode F. The latter are needed to formulate the initial conditions for the problem describing excitation and amplification of unstable mode $\mathrm{S}$ in the downstream region. It should be noted that the transition onset point $x_{t r}$ weakly (logarithmically) depends on the initial amplitude $A_{0}$ of instability. Approximate modelling of $A_{0}$ is, as a rule, sufficient to predict $x_{t r}$ with the accuracy expectable for practice.

The second series of DNSs was carried out for more realistic cases where the temperature spots were present in the free stream. These spots propagate downstream and pass through the bow shock induced by the viscous-inviscid interaction. It was shown that the spot-shock interaction plays a crucial role in the receptivity process. The dominant excitation of unstable waves is governed by the two-step mechanism. First, the spots interact with the bow shock and generate acoustic waves. Second, the induced acoustic waves penetrate into the boundary layer and generate unstable waves. Even in the case of weak shock considered herein, this mechanism provides the instability amplitudes two order of magnitude higher than in the case of receptivity to the temperature spottiness itself. Presumably, a similar mechanism drives receptivity to free-stream turbulence associated with vortical disturbances. Although there are no acoustic sources in the free flight, the interaction of free-stream disturbances with the bow shock may provide a sufficiently 'noisy' near field to trigger the transition process. Unfortunately it is not clear on this stage how to model the bow shock effect theoretically. Presumably, some progress could be made using the theory of Djakov (1957) and McKenzie \& Westphal (1968) to predict the induced acoustic field behind the shock and the asymptotic theory of Fedorov \& Khokhlov (1991) to evaluate receptivity of the boundary-layer flow to this acoustic field. It is also interesting to seek for the leading-edge shapes minimizing the shock-induced noise in order to delay transition.

\section{Acknowledgements}

This work was supported by the Russian Government under grant 'Measures to Attract Leading Scientists to Russian Educational Institutions' (contract No. 11.G34.31.0072). The authors would like to thank Professor I. Egorov (Central Aerohydrodynamic Institute) for helpful discussions of numerical methods and the problem formulation.

\section{REFERENCES}

Bushnell, D. 1990 Notes on initial disturbance fields for the transition problem. In Instability and Transition (ed. M. Y. Hussaini \& R. G. Voigt), vol. I, pp. 217-232. Springer.

ChOudhari, M. 1994 Theoretical prediction of boundary-layer receptivity. AIAA paper 94-2223.

Choudhari, M. \& STREeTt, C. 1990 Boundary-layer receptivity phenomena in three-dimesnional and high-speed boundary layers. AIAA paper 90-5258.

DJAKOv, S. P. 1957 Interaction of shocks with small disturbances. J. Expl Theor. Phys. 33, 948-961 (in Russian).

Egorov, I. V., Fedorov, A. V. \& Soudakov, V. G. 2005 Direct numerical simulation of supersonic boundary-layer receptivity to acoustic disturbances. AIAA Paper 2005-97.

Egorov, I. V., Fedorov, A. V. \& Soudakov, V. G. 2006 Direct numerical simulation of disturbances generated by periodic suction-blowing in a hypersonic boundary layer. Theor. Comput. Fluid Dyn. 20 (1), 41-54.

Egorov, I. V., Fedorov, A. V. \& Soudakov, V. G. 2008 Receptivity of a hypersonic boundary layer over a flat plate with a porous coating. J. Fluid Mech. 601, 165-187. 
Fedorov, A. V. 2003 Receptivity of a high-speed boundary layer to acoustic disturbances. J. Fluid Mech. 491, 101-129.

Fedorov, A. 2011 Transition and stability of high-speed boundary layers. Annu. Rev. Fluid Mech. 43, 79-95.

Fedorov, A. V. \& Khokhlov, A. P. 1991 Excitation of unstable modes in supersonic boundary layer by acoustic waves. Fluid Dyn. 9, 456-467.

Fedorov, A. V. \& Khokhlov, A. P. 2001 Prehistory of instability in a hypersonic boundary layer. Theor. Comput. Fluid Dyn. 14 (6), 359-375.

Fedrorov, A. V. \& KozLov, M. V. 2011 Receptivity of high-speed boundary layer to solid particulates. AIAA Paper 2011-3925.

Fedorov, A. V. \& Tumin, A. M. 2003 Initial-value problem for hypersonic boundary-layer flows. AIAA J. 41 (3), 379-389.

FEdorov, A. \& Tumin, A. 2010 Branching of discrete modes in high-speed boundary layers and terminology issues. AIAA Paper 2010-5003.

Gaydos, P. \& TUMin, A. 2004 Multimode decomposition in compressible boundary layers. AIAA J. 42 (6), 1115-1121.

Goldstein, M. E. 1983 The evolution of Tollmien-Schlichting waves near a leading edge. J. Fluid Mech. 127, 59-81.

Goldstein, M. E. 1985 Scattering of acoustic waves into Tollmien-Schlichting waves by small streamwise variation in surface geometry. J. Fluid Mech. 154, 509-529.

Heitmann, D. \& Radespiel, R. 2011 Simulation of the interaction of a laser generated shock wave with a hypersonic conical boundary layer. AIAA Paper 2011-3875.

Heitmann, D., Radespiel, R. \& Knauss, H. 2011 Experimental study of Mach 6 boundary layer response to laser generated disturbances. AIAA Paper 2011-3876.

HUANG, Y. \& ZHONG, X. 2010 Numerical study of laser-spot effects on boundary-layer receptivity for blunt compression-cones in Mach-6 freestream. AIAA Paper 2010-4447.

Jaffe, N. A., OKamura, T. T. \& SMith, A. M. O 1970 Determination of spatial amplification factors and their application to predicting transition. AIAA J. 8 (2), 301-308.

JiANG, G.-S. \& SHU, C.-W. 1996 Efficient implementation of weighted ENO schemes. J. Comput. Phys. 126, 202-228.

KIMMEL, R. 2003 Aspects of hypersonic boundary layer transition control. AIAA Paper 2003-0772.

MA, Y. \& ZHONG, X. 2001 Numerical simulation of receptivity and stability of nonequilibrium reacting hypersonic boundary layers. AIAA Paper 2001-0892.

MA, Y. \& ZHONG, X. 2003 Receptivity of a supersonic boundary layer over a flat plate. Part 2. Receptivity to free-stream sound. J. Fluid Mech. 488, 79-121.

MA, Y. \& ZHONG, X. 2005 Receptivity of a supersonic boundary layer over a flat plate. Part 3. Effects of different types of free-stream disturbances. J. Fluid Mech. 532, 63-109.

MACK, L. M. 1969 Boundary layer stability theory. Part B. Doc. 900-277, JPL, Pasadena, California, May.

MALIK, M. R. 1997 Boundary-layer transition prediction toolkit. AIAA Paper 97-1904.

MALIK, M. R. \& BALAKUMAR, P. 2005 Receptivity of supersonic boundary layers to acoustic disturbances. AIAA Paper 2005-5027.

MALIK, M. R., ZANG, T. \& Bushnell, D. 1990 Boundary layer transition in hypersonic flows. AIAA Paper 90-5232.

Maslov, A. A., Shiplyuk, A. N., Sidorenko, A. \& Arnal, D. 2001 Leading-edge receptivity of a hypersonic boundary layer on a flat plate. J. Fluid Mech. 426, 73-94.

McKenZIE, J. F. \& WeStPhal, K. O. 1968 Interaction of linear waves with oblique shock waves. Phys. Fluids 11 (11), 2350-2362.

Reshotko, E. 2008 Transition issues for atmospheric entry. J. Spacecr. Rockets 45, 161-164.

RUBAN, A. I. 1984 On Tollmien-Schlichting waves generation by sound. Izv. Akad. Nauk SSSR Mekh. Zhidk. Gaza (6), 60-67.

SAlyer, T. R., Collicott, S. H. \& Schneider, S. P. 2006 Characterizing laser-generated hot spots for receptivity studies. AIAA J. 44 (12), 2871-2878.

Schmeisseur, J. D., SChneider, S. P. \& Collicott, S. H. 2002 Supersonic boundary-layer response to optically generated freestream disturbances. Exp. Fluids 33, 225-232. 
SChneider, S. P., Collicott, S. H. \& Schmeisseur, J. D. 2000 Laser-generated localized freestream perturbations in supersonic and hypersonic flows. AIAA J. 38 (4), 666-671.

Schneider, S. P., Wheaton, B. M., Julinao, T. J., Berridge, D. C., Chou, A., Gilbert, P. L., CASPER, K. M. \& STEEN, L. E. 2009 Instability and transition measurements in the Mach-6 quiet tunnel. AIAA Paper 2009-3559.

SoudAKOV, V. G. 2010 Numerical simulation of the effect of acoustic wave inclination angle on a hypersonic boundary-layer receptivity. TsAGI Sci. J. 41 (3), 269-284.

WU, X. 1999 Generation of Tollmien-Schlichting waves by convecting gusts interacting with sound. J. Fluid Mech. 397, 285-316.

Zhigulev, V. N. \& Fedorov, A. V. 1987 On boundary layer receptivity to acoustic disturbances. J. Prikl. Mekh. Tekh. Fiz. (6), 43-49.

Zhigulev, V. N. \& Tumin, A. M. 1987 Onset of Turbulence. Nauka, (in Russian).

ZHONG, X. 2001 Leading-edge receptivity to free-stream disturbance waves for hypersonic flow over parabola. J. Fluid Mech. 441, 315-367. 